\title{
Fast Algorithms for Generating Parametric Cubic Spline Interpolation Curves
}

This paper was downloaded from TechRxiv (https://www.techrxiv.org).

\section{LICENSE}

CC BY 4.0

SUBMISSION DATE / POSTED DATE

$10-02-2022$ / 16-02-2022

CITATION

LIAN, JIAN-AO (2022): Fast Algorithms for Generating Parametric Cubic Spline Interpolation Curves. TechRxiv. Preprint. https://doi.org/10.36227/techrxiv.19154132.v1

DOI

10.36227/techrxiv.19154132.v1 


\title{
Fast Algorithms for Generating Parametric Cubic Spline Interpolation Curves
}

\author{
Jian-ao Lian \\ Math. Dept. \\ Prairie View A\&M University \\ Prairie View, Texas, USA \\ unurbs@yahoo.com
}

\begin{abstract}
For any given two or three dimensional dataset as an open or closed piecewise linear function, two algorithms are established for constructing geometrically differentiable parametric cubic splines that interpolate the given dataset. These algorithms do not require any additional tangent direction information at each datapoint or any boundary conditions. One algorithm is for the local least square parametric cubic spline interpolation so that a given dataset can be best approximated through appropriately defined local least squares approximation. Simple examples will be used to illustrate the algorithms and various datasets will also be demonstrated to show the efficiency of the algorithms.
\end{abstract}

Keywords-Computer graphics, interpolation, least squares approximation, line and curve generation, point cloud, spline and piecewise polynomial

\section{INTRODUCTION}

Cubic splines, or piecewise cubic polynomials (PCP), have been widely used for data interpolation and computer graphics for decades. Spline curves and surfaces provide convenience for repositioning some control points in order to restructure the shape of the curve or surface. First of all, such reposition of control points or shape modification requires locality, meaning not causing any global change. Second, to adapt to arbitrary data, it is imperative that parametric cubic splines are exploited. There are a few classical algorithms for two-dimensional (2d) curve design in the literature. Two widely used ones are the de Casteljau algorithm and Chaikin's corner-cutting algorithm, though both algorithms were easily extended to three-dimensional (3d) settings. The former one, cf., e.g., [14, p. 13], was for evaluating values on polynomials generated by recursive interpolations of line segments. These line segments are continuously connected by prescribed $2 \mathrm{~d}$ or $3 \mathrm{~d}$ points, called control points. The latter one, a $2 \mathrm{~d}$ or $3 \mathrm{~d}$ subdivision scheme, cf., e.g., [4], can generate smooth curves from a set of initial control points. However, both algorithms do not interpolate all given points. Furthermore, though there were some studies of limiting curves of subdivision schemes in terms of refinable functions, cf., e.g., [11], the expressions for the final piecewise polynomials through subdivision schemes are still not so explicit.

Univariate and multivariate splines have been consistently studied ( [3], [5], [10], [15], [16]), and have been also successfully applied in various areas. These areas include, but are not limited to, curve reconstruction on a road map with data points and driving directions [1], machine learning (ML) [2], reliability approximation of a two-terminal network [6], energy optimization of Internet of Things (IoT) devices [7], developing new algorithms for VLSI implementation [8], processing and smoothing one-dimensional signals and experimental data in digital signal processors [9], generation of uniform cubic $B$-spline interpolating data points under tangent and curvature vectors constraints [12], establishing a hybrid fuzzy logic-based intensity mapping for image enhancement [13], for image interpolation with prescribed tangents [17], and for better reconstructed image [18].

The main objective of this paper is to establish explicit parametric cubic spline interpolation curves (PACSIC) by using only the vertices on given piecewise linear functions (PLF). A PLF is either in $2 \mathrm{~d}$ or $3 \mathrm{~d}$ (and can be extended to any dimension), and either open or closed. The algorithms are local, meaning removing or inserting new interpolation points only causes curve change locally. In addition, least squares (LS) are utilized when the local square-integral is minimized to avoid solving a global least square problem. For an easy presentation, some preliminaries will be constituted in Section II; efficient algorithms for $G^{1}$ PACSIC and LS $G^{1}$ PACSIC will be established in Section III and Section IV A $G^{1}$ PACSIC or LS $G^{1}$ PACSIC created from the two algorithms, or a $G^{2}$ PACSIC, or any collection of PCP, can be stored in a pseudo PLY formatted file, which will be presented in Section $\mathrm{V}$, followed by illustrations of the algorithms in Section VI. Some simple applications will be demonstrated in Section VII Similar study can be carried out to $G^{2}$ PACSIC, which will be briefly touched upon in Section VIII, with all $G^{2}$ conditions being specified in terms of elastic parameters. The conclusion will be comprised in Section IX Two lemmas for formulas used for both algorithms will be enclosed and proved in Appendix A and Appendix B.

\section{PRELIMINARIES}

An $n$-dimensional ( $n$-d) degree $m$ Bézier curve, denoted by $\mathbf{B}(t)=\left[B_{1}(t), \cdots, B_{n}(t)\right]^{\top}$, is defined by

$$
\mathbf{B}(t)=\sum_{k=0}^{m} \mathbf{c}_{k} b_{m, k}(t), \quad t \in[0,1]
$$


where $\mathbf{c}_{0}, \cdots, \mathbf{c}_{m} \in \mathbb{R}^{n}$, are called control points, with

$$
b_{m, k}(t)=\left(\begin{array}{c}
m \\
k
\end{array}\right) t^{k}(1-t)^{m-k}, k=0, \ldots, m,
$$

being the Bézier basis or Bernstein polynomials. $\mathbf{B}(t)$ in 11 interpolates tangents as well as values at the first and last control points, namely:

$$
\begin{aligned}
& \mathbf{B}(0)=\mathbf{c}_{0}, \quad \mathbf{B}(1)=\mathbf{c}_{m} ; \quad \text { and } \\
& \mathbf{B}^{\prime}(0)=m\left(\mathbf{c}_{1}-\mathbf{c}_{0}\right), \quad \mathbf{B}^{\prime}(1)=m\left(\mathbf{c}_{m}-\mathbf{c}_{m-1}\right) .
\end{aligned}
$$

Let $\mathbf{B}_{1}(t)$ and $\mathbf{B}_{2}(t)$ be two Bézier curves:

$$
\begin{array}{ll}
\mathbf{B}_{1}(t)=\sum_{k=0}^{m} \mathbf{c}_{1, k} b_{m, k}(t), & t \in[0,1], \\
\mathbf{B}_{2}(t)=\sum_{k=0}^{m} \mathbf{c}_{2, k} b_{m, k}(t), & t \in[0,1] .
\end{array}
$$

All continuity conditions, when $\mathbf{B}_{1}(t)$ and $\mathbf{B}_{2}(t)$ are connected, can be expressed exactly by their control points $\mathbf{c}_{1, k}$ and $\mathbf{c}_{2, k}, k=0, \ldots, m$. For parametric curves, though the $G^{0}$ and $G^{1}$ continuities are more appropriate and will be the main objective, $G^{2}$ continuity will also be embraced. $G^{0}$ continuity is the normal $C^{0}$ continuity. However, $G^{1}$ continuity means two curves share a common tangent direction at their joint point. First, $\mathbf{B}_{1}(t)$ and $\mathbf{B}_{2}(t)$ in $\sqrt{2}-(3)$ are $G^{0}$ connected if and only if $\mathbf{B}_{1}(1)=\mathbf{B}_{2}(0)$, i.e.,

$$
\mathbf{c}_{2,0}=\mathbf{c}_{1, m} .
$$

Second, the $G^{1}$ continuity between $\mathbf{B}_{1}(t)$ and $\mathbf{B}_{2}(t)$ requires both (4) and $\mathbf{B}_{2}^{\prime}(0)=\xi \mathbf{B}^{\prime}{ }_{1}(1)$ for some $\xi \in \mathbb{R}$, which is

$$
\mathbf{c}_{2,1}-\mathbf{c}_{2,0}=\xi\left(\mathbf{c}_{1, m}-\mathbf{c}_{1, m-1}\right) \text {. }
$$

Observe that the $G^{1}$ conditions in 4 - -5 indicate that the three vectors $\mathbf{c}_{1, m-1}, \mathbf{c}_{1, m}=\mathbf{c}_{2,0}$, and $\mathbf{c}_{2,1}$ are collinear.

Set $m=3$ in the sequel; and focus on $G^{1}$ PACSIC first. To facilitate an easy presentation, let

$$
\mathbf{P}_{1}, \ldots, \mathbf{P}_{n},
$$

be the $(2 \mathrm{~d}$ or $3 \mathrm{~d})$ interpolation data points, and

$$
\mathbf{B}(t)=\left[\mathbf{B}_{1}(t), \cdots, \mathbf{B}_{n-1}(t)\right],
$$

be the PACSIC that interpolates $\mathbf{P}_{i}, i=1, \ldots, n$. More precisely, write

$$
\begin{aligned}
& \mathbf{B}_{i}(t)=\mathbf{P}_{i} b_{3,0}(t)+\mathbf{Q}_{i, 1} b_{3,1}(t) \\
& \quad+\mathbf{Q}_{i, 2} b_{3,2}(t)+\mathbf{P}_{i+1} b_{3,3}(t), i=1, \ldots, n-1 .
\end{aligned}
$$

where, $\mathbf{B}_{i}(t)$ interpolates $\mathbf{P}_{i}$ and $\mathbf{P}_{i+1}$ at its two endpoints, and $\mathbf{Q}_{i, 1}$ and $\mathbf{Q}_{i, 2}$ are $\mathbf{B}_{i}(t)$ 's two interior control points. By doing so, $\mathbf{B}_{1}(t), \ldots, \mathbf{B}_{n-1}(t)$ are continuously connected; and all $G^{1}$ conditions 4 - 5 between adjacent PACSIC in (7) become

$$
\mathbf{Q}_{i+1,1}-\mathbf{P}_{i+1}=\xi_{i}\left(\mathbf{P}_{i+1}-\mathbf{Q}_{i, 2}\right), \quad i=1, \ldots, n-1,
$$

for some nonzero constants $\xi_{1}, \ldots, \xi_{n-1} \in \mathbb{R}$.

\section{Algorithm FOR $G^{1}$ PACSIC}

There are a plenty of $G^{1}$ PACSIC curves that interpolate $\mathbf{P}_{i}$ 's in (6), as it will be shown in this section. An efficient algorithm of producing such an curve is given in the following.

\section{Algorithm 1}

1) The tangent direction at each interior knot is determined to be parallel to the line passing its two neighbors:

$$
\begin{aligned}
\mathbf{Q}_{i-1,2} & =\mathbf{P}_{i}-\alpha_{i}\left(\mathbf{P}_{i+1}-\mathbf{P}_{i-1}\right), \\
\mathbf{Q}_{i, 1} & =\mathbf{P}_{i}+\beta_{i}\left(\mathbf{P}_{i+1}-\mathbf{P}_{i-1}\right),
\end{aligned}
$$

for $i=2, \ldots, n-1$, where $\alpha_{i}$ and $\beta_{i}$ are (positive) elastic parameters. Fig. 1 illustrates how the new (local) control points are created.

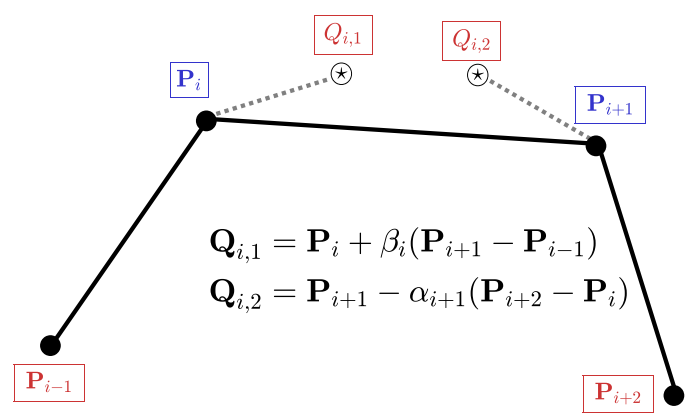

Fig. 1: New control points are introduced locally.

2) If the initial PLF is open, namely: $\mathbf{P}_{n} \neq \mathbf{P}_{1}$,

$$
\begin{aligned}
\mathbf{Q}_{1,1} & =\mathbf{P}_{1}+\beta_{1}\left(\mathbf{P}_{2}-\mathbf{P}_{1}\right), \\
\mathbf{Q}_{n-1,2} & =\mathbf{P}_{n}-\alpha_{n}\left(\mathbf{P}_{n}-\mathbf{P}_{n-1}\right) ;
\end{aligned}
$$

and if $\mathbf{P}_{n}=\mathbf{P}_{1}$,

$$
\begin{aligned}
\mathbf{Q}_{1,1} & =\mathbf{P}_{1}+\beta_{1}\left(\mathbf{P}_{2}-\mathbf{P}_{n-1}\right), \\
\mathbf{Q}_{n-1,2} & =\mathbf{P}_{1}-\alpha_{n}\left(\mathbf{P}_{2}-\mathbf{P}_{n-1}\right) .
\end{aligned}
$$

3) With all pairs of $\left(\mathbf{Q}_{1,1}, \mathbf{Q}_{1,2}\right), \ldots,\left(\mathbf{Q}_{n-1,1}, \mathbf{Q}_{n-1,2}\right)$ well defined, i.e., from (8)-11) for open PLF and (8)(9) and (12)-13 for closed PLF, the $n-1$ or $n$ piecewise bicubic polynomials $\left\{\mathbf{B}_{i}\right\}_{i=1, \ldots, n-1}$ or $\left\{\mathbf{B}_{i}\right\}_{i=1, \ldots, n}$ are determined from $n-1$ or $n$ sets of quadruple control points

$$
\left\{\mathbf{P}_{i}, \quad \mathbf{Q}_{i, 1}, \quad \mathbf{Q}_{i, 2}, \quad \mathbf{P}_{i+1}\right\}
$$

for $i=1, \ldots, n-1$.

Observe that the two tangent directions in (8)- 9 over each line segment are local, or the two new control points $\mathbf{Q}_{i, 1}$ and $\mathbf{Q}_{i, 2}$ only depend on the four nearby initial vertices $\mathbf{P}_{i-1}$, $\mathbf{P}_{i}, \mathbf{P}_{i+1}$, and $\mathbf{P}_{i+2}$. In other words, adding, removing, or repositioning a vertex only affects the $G^{1}$ PACSIC locally. It is also worthwhile to point out that $\alpha_{2}, \ldots, \alpha_{n}$ and $\beta_{1}, \ldots$, $\beta_{n-1}$, in (8) -9) are the elastic parameters, where neither $\alpha_{1}$ nor $\beta_{n}$ is defined. However, if the initial PLF is closed, define $\alpha_{1}=\alpha_{n}$ and $\beta_{n}=\beta_{1}$. 


\section{Algorithm FOR LS $G^{1}$ PACSIC}

Among all $G^{1}$ PACSIC curves in Algorithm 1 in Section III. there is a unique curve that is the LS approximation to the piecewise linear polynomial formed by the data points $\mathbf{P}_{1}, \ldots, \mathbf{P}_{n}$. For LS, the Euclidean norm is used, e.g., $\left\|\mathbf{B}_{i}(t)\right\|^{2}=\mathbf{B}_{i}(t)^{\star} \mathbf{B}_{i}(t)$, where $\star$ denotes the transpose of the complex conjugate.

The LS is defined in the following algorithm and is used for determining the elastic parameters for LS PACSIC.

\section{Algorithm 2}

1) Write $\mathbf{Q}_{i-1,2}$ and $\mathbf{Q}_{i, 1}$ the same as in (8)-(9), for $i=2$, $\ldots, n-1 ; \mathbf{Q}_{1,1}$ and $\mathbf{Q}_{n-1,2}$ the same as in either (10)(11) or (12)-(13) depending on if the initial PLF is open or closed.

2) Evaluate the elastic parameter $\alpha_{i+1}$ and $\beta_{i}, i=2, \ldots$, $n-2$, in $\mathbf{Q}_{i, 1}$ given in $(9)$ and $\mathbf{Q}_{i, 2}$ in $(8)$ with $i$ replaced by $i+1$, by

$$
\begin{gathered}
\beta_{i}=\frac{4\left\|\mathbf{e}_{i}\right\|^{2} \mathbf{e}_{i-1}^{\top} \mathbf{d}_{i}+3 \mathbf{e}_{i}^{\top} \mathbf{e}_{i-1} \mathbf{e}_{i}^{\top} \mathbf{d}_{i}}{3\left[16\left\|\mathbf{e}_{i-1}\right\|^{2}\left\|\mathbf{e}_{i}\right\|^{2}-9\left(\mathbf{e}_{i-1}^{\top} \mathbf{e}_{i}\right)^{2}\right]}, \\
\alpha_{i+1}=\frac{4\left\|\mathbf{e}_{i-1}\right\|^{2} \mathbf{e}_{i}^{\top} \mathbf{d}_{i}+3 \mathbf{e}_{i-1}^{\top} \mathbf{e}_{i} \mathbf{e}_{i-1}^{\top} \mathbf{d}_{i}}{3\left[16\left\|\mathbf{e}_{i-1}\right\|^{2}\left\|\mathbf{e}_{i}\right\|^{2}-9\left(\mathbf{e}_{i-1}^{\top} \mathbf{e}_{i}\right)^{2}\right]},
\end{gathered}
$$

where $\mathbf{e}_{i-1}, \mathbf{e}_{i}$, and $\mathbf{d}_{i}$ are the three directions

$$
\begin{aligned}
& \mathbf{e}_{i-1}=\mathbf{P}_{i+1}-\mathbf{P}_{i-1}, \\
& \mathbf{e}_{i}=\mathbf{P}_{i+2}-\mathbf{P}_{i}, \\
& \mathbf{d}_{i}=\mathbf{P}_{i+1}-\mathbf{P}_{i} .
\end{aligned}
$$

Here the deteriorate scenario of either $\mathbf{P}_{i-1}=\mathbf{P}_{i+1}$ or $\mathbf{P}_{i}=\mathbf{P}_{i+2}$ was excluded. The proof of $(14)-(15)$ is prolonged to Lemma 2 in Appendix A.

3) If the inital PLF is closed, i.e, $\mathbf{P}_{n}=\mathbf{P}_{1}$, define $\mathbf{P}_{0}$, and $\mathbf{P}_{n+1}$ periodically, i.e., $\mathbf{P}_{0}=\mathbf{P}_{n-1}$, and $\mathbf{P}_{n+1}=\mathbf{P}_{2}$; if the PLF is open, define $\mathbf{P}_{0}=\mathbf{P}_{1}$ and $\mathbf{P}_{n+1}=\mathbf{P}_{n}$.

4) For the PCP over the first segment $\mathbf{P}_{1} \rightarrow \mathbf{P}_{2}$, with $\mathbf{Q}_{1,1}$ in either (10) or (12) and $\mathbf{Q}_{1,2}$ in (8) with $i=2$, evaluate $\left(\beta_{1}, \alpha_{2}\right)$ via $(14)-(18)$ with $i=1$.

5) For the PCP over the last segment $\mathbf{P}_{n-1} \rightarrow \mathbf{P}_{n}$, with $\mathbf{Q}_{n-1,1}$ in 97 for $i=n-1$ and $\mathbf{Q}_{n-1,2}$ in either (11) or (13), evaluate $\left(\beta_{n-1}, \alpha_{n}\right)$ via (14)-18) with $i=n-1$. At this point, all elastic parameters $\alpha_{2}, \ldots, \alpha_{n}$ and $\beta_{1}$, $\ldots, \beta_{n-1}$ for the LS PACSIC are evaluated.

\section{PACSIC FILE FORMAT}

As soon as the two new control points for each PCP in a PACSIC determined in both algorithms in Section refalgorithm1 and Section IV] the PACSIC is completely determined. This PACSIC can be stored in a pseudo PLY file in the following, with its header ommited.

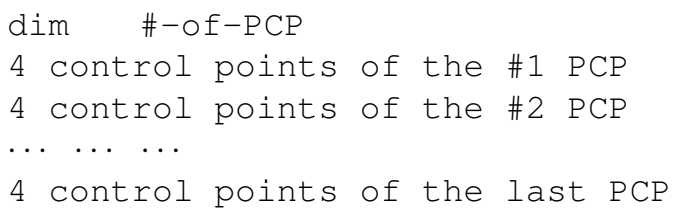

For instance, the PLY file

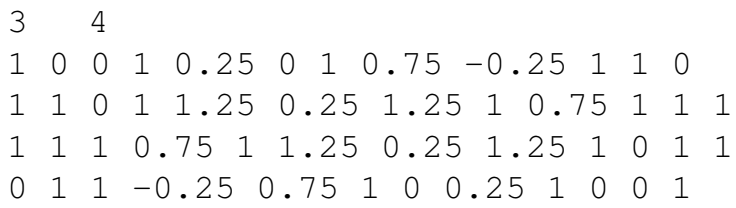

is a PACSIC for four $3 \mathrm{~d}$ PCP connected in the $G^{1}$ fashion, which plotted in Fig. 2

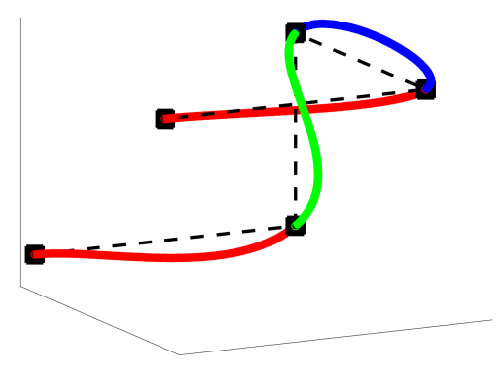

Fig. 2: A pseudo PLY file is plotted, which is a $G^{1}$ PACSIC created from a PLF through 5 vertices on a cube. The PLY file stored all data for the PACSIC: a collection of four 3d PCP.

However, such a file can also be a collection of any connected or disconnected PCP, $G^{1}$ or not $G^{1}$. E.g., the following PLY file

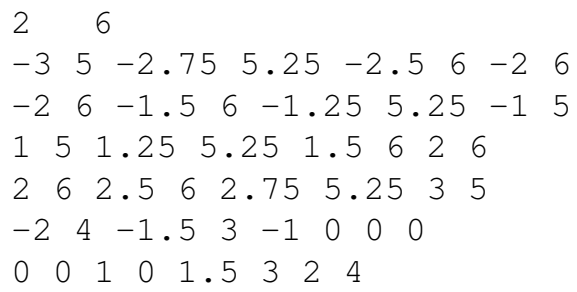

bears three 2d PACSIC plotted in Fig. 3

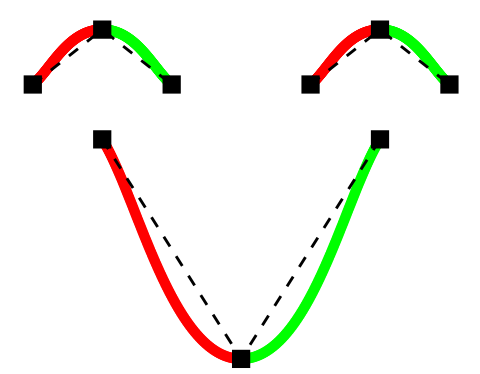

Fig. 3: A pseudo PLY file for a combination of 3 disjoint $2 \mathrm{~d}$ $G^{1}$ PACSIC created from 3 PLF.

\section{Algorithm Illustrations}

Some simple $2 \mathrm{~d}$ and $3 \mathrm{~d}$ sample datasets are being used in this section to illustrate Algorithm 1 in Section IIII \& Algorithm 2 in Section IV 
Illustration 1: Three 2d points for a two-side open PLF.

Given the three-point $2 \mathrm{~d}$ ordered dataset

$$
\mathbf{P}_{1}=\left[\begin{array}{l}
0 \\
0
\end{array}\right], \quad \mathbf{P}_{2}=\left[\begin{array}{l}
2 \\
2
\end{array}\right], \quad \mathbf{P}_{3}=\left[\begin{array}{l}
3 \\
0
\end{array}\right]
$$

Fig. 4 shows the $G^{1}$ PACSIC by using Algorithm 1 with fixed uniform elastic parameters 0.25 , where each end vertex was used twice.

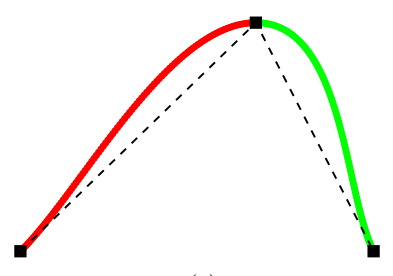

(a)

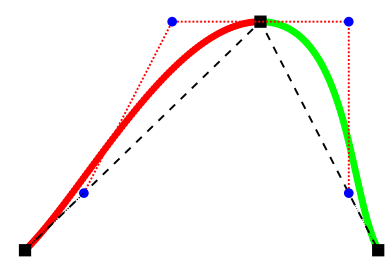

(b)
Fig. 4: (a) $G^{1}$ PACSIC with dataset in 19 with global elastic parameter 0.25, where the initial open PLF is in black dashed lines; and (b) the 2 pairs of new control points (in blue) for the two PCP of the $G^{1}$ PACSIC are included.

Illustration 2: Four 2d points for a closed PLF (or a triangle).

Extend the open PLF in (19) to be closed as a triangle:

$$
\mathbf{P}_{1}=\left[\begin{array}{l}
0 \\
0
\end{array}\right], \quad \mathbf{P}_{2}=\left[\begin{array}{l}
2 \\
2
\end{array}\right], \quad \mathbf{P}_{3}=\left[\begin{array}{l}
3 \\
0
\end{array}\right], \quad \mathbf{P}_{4}=\left[\begin{array}{l}
0 \\
0
\end{array}\right],
$$

Fig. 5a shows the $G^{1}$ PACSIC by using Algorithm 1 with fixed uniform elastic parameters 0.25 . The closed PLF is convex and so is the PACSIC when the global elastic parameter is chosen to be between 0 and 1. Fig. $5 b$ also illustrates how the new control points were created: the tangent direction at each vertex is parallel to the line formed by the remaining two vertices.

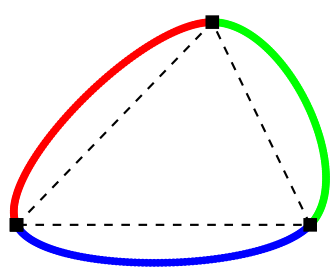

(a)

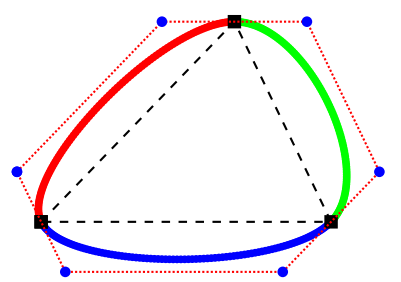

(b)
Fig. 5: (a) $G^{1}$ PACSIC with dataset in 20) with global elastic parameter 0.25 . (b) The 3 pairs of new control points (in blue color) for the three PCP of the $G^{1}$ PACSIC are included.

Illustration 3: LS PACSIC for a triangle.

Again, with the same four-point $2 \mathrm{~d}$ dataset in 20, Fig. 6a shows the LS $G^{1}$ PACSIC by applying Algorithm 2, where the new pairs of the two middle control points are listed in the following.

$$
\begin{aligned}
& {\left[\mathbf{Q}_{1,1}, \mathbf{Q}_{1,2}, \mathbf{Q}_{2,1}, \mathbf{Q}_{2,2}, \mathbf{Q}_{3,1}, \mathbf{Q}_{3,2}\right]} \\
& =\left[\begin{array}{cccccc}
-\frac{2}{213} & \frac{392}{213} & \frac{143}{69} & \frac{208}{69} & \frac{436}{151} & \frac{5}{151} \\
\frac{4}{213} & 2 & 2 & \frac{1}{69} & -\frac{17}{151} & -\frac{10}{151}
\end{array}\right] .
\end{aligned}
$$

Observe that, the uniqueness of the LS PACSIC is due to the unique solution for all elastic parameters from Algorithm 2. Though it does look like it is not $G^{1}$ at the three vertices, the LS PACSIC in Fig. 6 are indeed $G^{1}$.

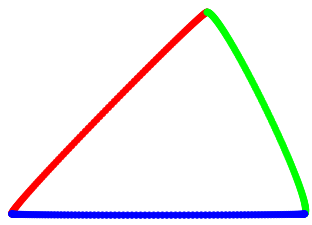

(a)

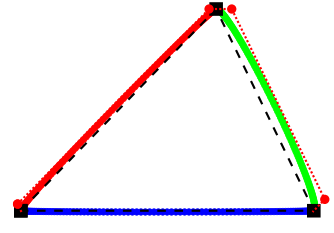

(b)
Fig. 6: (a) LS $G^{1}$ PACSIC with dataset in 20; and (b) the 3 pairs of new control points (in red), given in 21, for the three PCP of the LS $G^{1}$ PACSIC are included.

Illustration 4: A 3d seven point closed PLF.

Both algorithms also word for 3d PLF. Fig. 7 shows the $G^{1}$ PACSIC for seven points of the $3 \mathrm{~d}$ closed PLF, listed in the following as a matrix

$$
\left[\mathbf{P}_{1}, \cdots, \mathbf{P}_{7}\right]=\left[\begin{array}{ccccccc}
0 & 1 & 1 & 1 & 0 & 0 & 0 \\
0 & 0 & 1 & 2 & 2 & 1 & 0 \\
-1 & 1 & -2 & 3 & -3 & 1 & -1
\end{array}\right] .
$$

Algorithm 1 was first applied with fixed uniform elastic parameters 0.25 . New control points as well as the seven vertices of the original closed 3d PLF are included in Fig. $7 b$ For comparison purposes, the LS PACSIC is provided in Fig. $7 \mathrm{c}$

\section{Algorithm Demonstrations}

A few $2 \mathrm{~d}$ and $3 \mathrm{~d}$ datasets are being used in this section to demonstrate the applications of Algorithm 1 in Section III \& Algorithm 2 in Section IV to various datasets. In addition, though the elastic parameters $\alpha_{i}$ and $\beta_{i}$ in (8)-(9) can in general be arbitrarily chosen to be on the interval $(0,1)$, they are not necessarily uniformly selected as seen for LS PACSIC in Algorithm 2. Indeed, other than the LS PACSIC, these elastic parameters can be any real number, e.g., $\alpha_{i}, \beta_{i}<0$ or $\alpha_{i}, \beta_{i}>1$.

Demonstration 1: Repeated datapoint.

By using the four-point $2 \mathrm{~d}$ dataset in 20 but letting the point $\mathbf{P}_{2}$ repeat twice and three times, Fig. 8 shows the $G^{1}$ PACSIC by using Algorithm 1 with fixed uniform elastic 


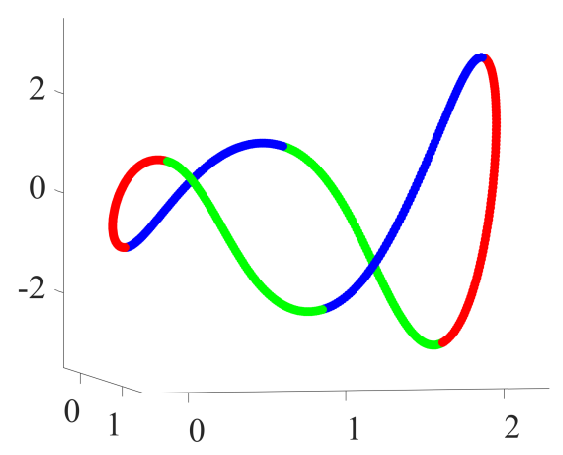

(a)

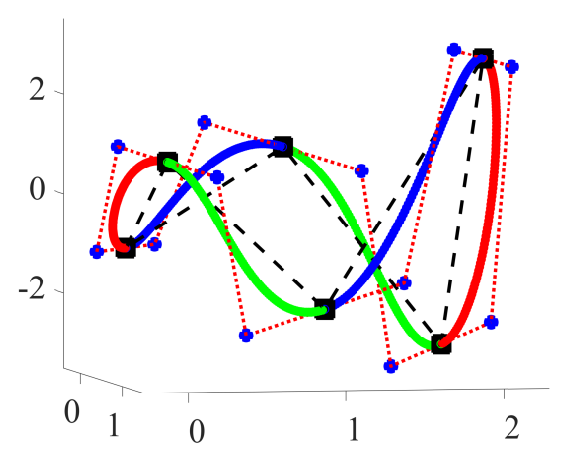

(b)

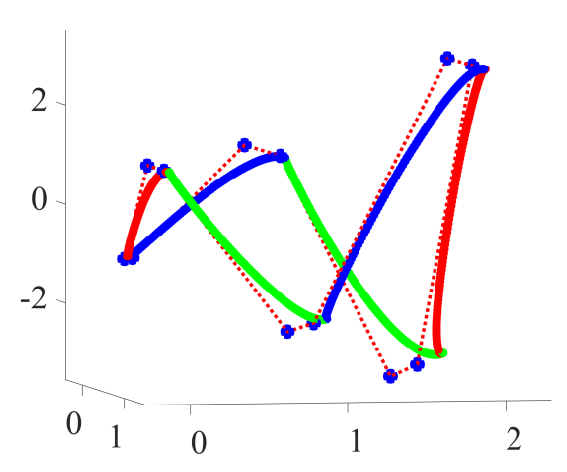

(c)

Fig. 7: $G^{1}$ PACSIC with 3d dataset in 22): (a) with global elastic parameter 0.25 ; (b) added the 6 pairs of new control points to (a), in blue color, for the six PCP of the PACSIC; (c) the LS PACSIC with new control points included.

parameters 0.25 . This demonstration reflects the consequences of repeating vertices. Certainly, there is still a unique solution for the LS PACSIC if $\mathbf{P}_{2}$ is repeated only twice (its graph is omitted here). However, there is no solution for LS PACSIC if $\mathbf{P}_{2}$ is repeated three or more times due to the fact that the necessary conditions (36), in Appendix A, are not satisfied.

Demonstration 2: Selection of elastic parameters.

Again, with the four-point $2 \mathrm{~d}$ dataset in (20), Fig. 9 shows the $G^{1}$ PACSIC by using Algorithm 1 with various selections for the elastic parameters $\alpha_{i}$ and $\beta_{i}, i=1,2,3$. In general, a

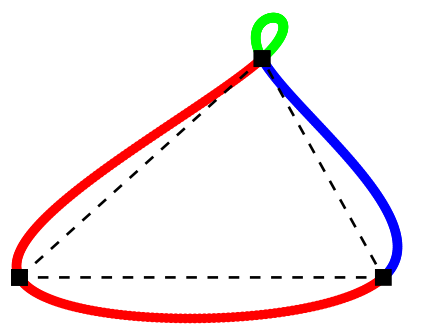

(a)

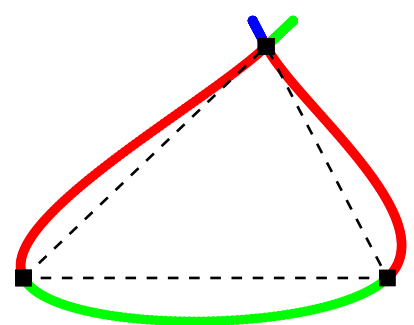

(b)
Fig. 8: $G^{1}$ PACSIC for dataset in 20) with global elastic parameter 0.25 , and 1 vertex $\mathbf{P}_{2}$ being appeared in the dataset (a) twice; and (b) three times.

$G^{1}$ PACSIC behaves well if (1) all elastic parameters are on $(0,1)$; or (2) the two elastic parameters at a vertex are both negative; or (3) some of them are $>1$. But when the two elastic parameters at a vertex have opposite signs, the resulting PACSIC is not really $G^{1}$, though the tangents at this vertex are collinear but they are along opposite directions.

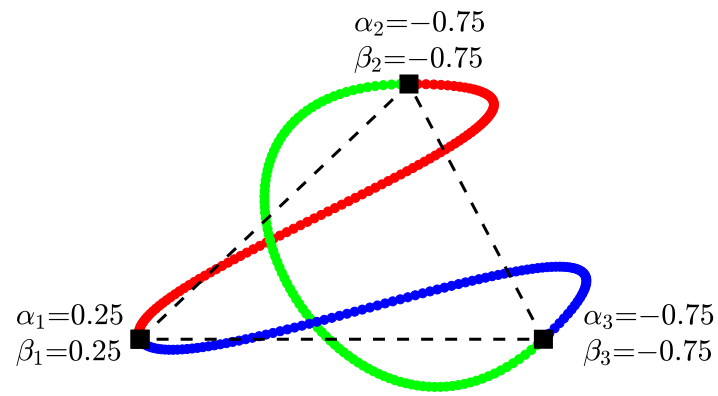

(a)

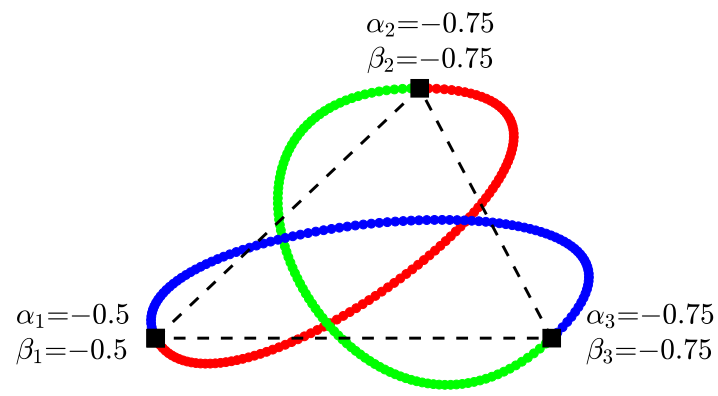

(b)

Fig. 9: $G^{1}$ PACSIC with dataset in 20 using two different sets of selections for the elastic parameters on each vertex.

Demonstration 3: Approximation to step functions.

Situate the dataset as the 10 vertices from the $M$-shaped step function, being also listed as a matrix

$$
\begin{aligned}
& {\left[\mathbf{P}_{1}, \mathbf{P}_{2}, \cdots, \mathbf{P}_{10}\right]} \\
& =\left[\begin{array}{llllllllll}
0 & 1 & 1 & 2 & 2 & 3 & 3 & 4 & 4 & 5 \\
0 & 0 & 1 & 1 & 0 & 0 & 1 & 1 & 0 & 0
\end{array}\right] .
\end{aligned}
$$

Fig. 10a shows the PACSIC with 0.25 as the uniform elastics parameter and its LS PACSIC in Fig. $10 \mathrm{~b}$ This means that any 
2d, 3d, or any dimensional PLF, e.g., the PLP with $m$ adjacent vertices in $\left\{\mathbf{v}_{1}, \ldots, \mathbf{v}_{m}\right\}$, can always be expressed by a unique $G^{1}$ PACSIC, provided that $\mathbf{v}_{i} \neq \mathbf{v}_{i+2}, i=1, \ldots, n-2$ (see (36) in Appendix A). In other words, the LS PACSIC can be a good alternative for any PLF. Furthermore, the unique LS PACSIC depends on the reference curve (see Appendix B). In general, the reference curve for a PCP over two adjacent vertices $\mathbf{P}_{i} \rightarrow \mathbf{P}_{i+1}$ is simply the line passing the two vertices (see (34) in Appendix A).

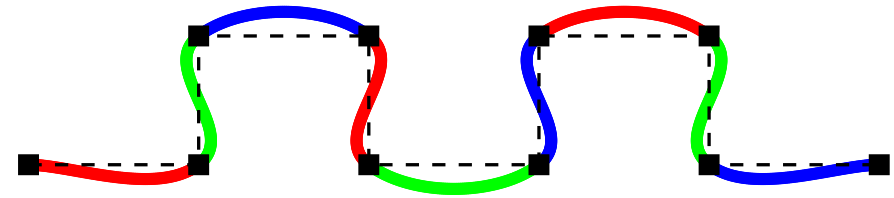

(a)

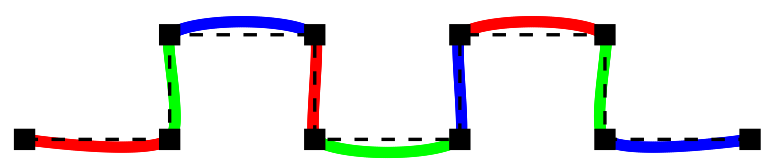

(b)

Fig. 10: (a) $G^{1}$ PACSIC with global elastic parameter 0.25 from dataset in 23 ; and (b) its LS $G^{1}$ PACSIC.

Demonstration 4: Approximation to a cross PLF.

Set the dataset as vertices for the 13 point cross-shaped closed PLF, i.e.,

$$
\begin{aligned}
& \left\{\mathbf{P}_{1}, \mathbf{P}_{2}, \cdots, \mathbf{P}_{13}\right\} \\
& =\left\{\left[\begin{array}{l}
0 \\
0
\end{array}\right],\left[\begin{array}{l}
1 \\
0
\end{array}\right],\left[\begin{array}{l}
1 \\
1
\end{array}\right],\left[\begin{array}{l}
2 \\
1
\end{array}\right],\left[\begin{array}{c}
2 \\
0
\end{array}\right],\left[\begin{array}{l}
3 \\
0
\end{array}\right],\left[\begin{array}{c}
3 \\
-1
\end{array}\right],\right. \\
& \left.\left[\begin{array}{c}
2 \\
-1
\end{array}\right],\left[\begin{array}{c}
2 \\
-2
\end{array}\right],\left[\begin{array}{c}
1 \\
-2
\end{array}\right],\left[\begin{array}{c}
1 \\
-1
\end{array}\right],\left[\begin{array}{c}
0 \\
-1
\end{array}\right],\left[\begin{array}{l}
0 \\
0
\end{array}\right]\right\} .
\end{aligned}
$$

Fig. 11 corroborates the LS behavior, where it shows a PACSIC to the dataset in 24) and with global elastic parameter 0.5 in Fig. 11a while its LS PACSIC is in Fig. 11b Both algorithms handle any closed PLF well.

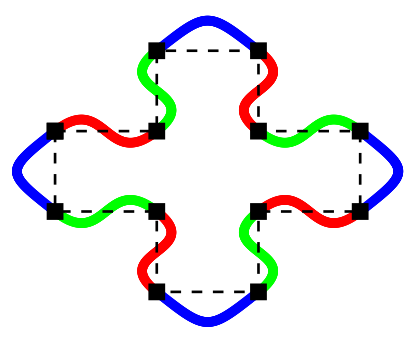

(a)

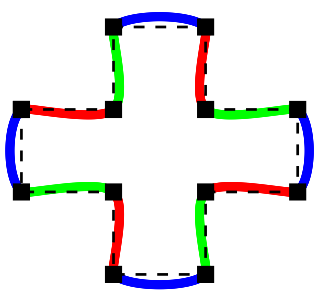

(b)
Fig. 11: $G^{1}$ PACSIC with global elastic parameter 0.5 and LS $G^{1}$ PACSIC from dataset in 24).

Demonstration 5: Four random PLF in a cube and LS PACSIC for a PLF on the unit sphere.
Fig. 12a is a set of 4 PACSIC created from 4 closed PLF in the unit cube in the first quadrant. The 4 PLF were randomly formed and passing the 4 pairs of vertices $\{[0,0,0],[1,1,1]\}$, $\{[1,0,0],[0,1,1]\},\{[0,1,0],[1,0,1]\}$, and $\{[1,1,0],[0,0,1]\}$, respectively.

Fig. $12 \mathrm{~b}$ shows the image after Algorithm 2 was applied to a closed PLF formed by 37 points on the unit sphere. The PACSIC is $G^{1}$ but all points on the PACSIC are not necessarily on the sphere.

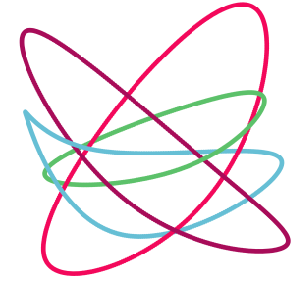

(a)

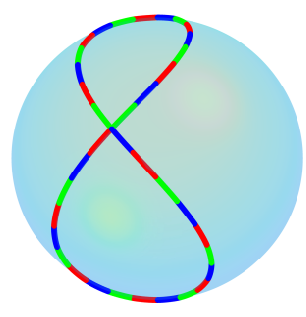

(b)
Fig. 12: (a) Four 3d $G^{1}$ PACSIC created from 4 randomly created closed PLF within the unit cube in the first quadrant; (b) the LS $G^{1}$ PACSIC for a closed 3d 37-point PLF on the unit sphere.

\section{Demonstration 6: A sequence of PACSIC.}

With the same dataset in (24), apply a sequence of Algorithm 1 for 20 values of the uniform elastic parameters $\alpha_{i}=\beta_{i}$ from 0.05 to 1 with an increment of 0.05 . Fig. 13a shows the image covered by the 20 PACSIC. Hence, for a given open or closed 3d PLF, it can be expanded to a point cloud with any desirable number of points, as shown in Fig. $13 \mathrm{~b}$ where Algorithm 1 was applied to the same 5 points on a cube, see Fig. 2. 61 times for a family of elastic parameters ranging from -1.5 to 1.5 with stepsize 0.05 .

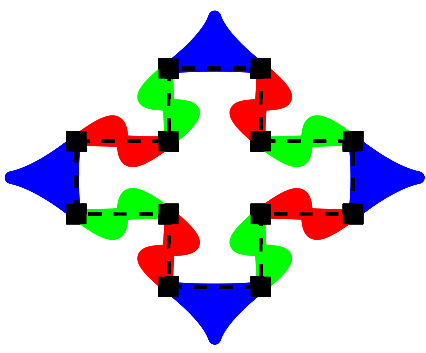

(a)

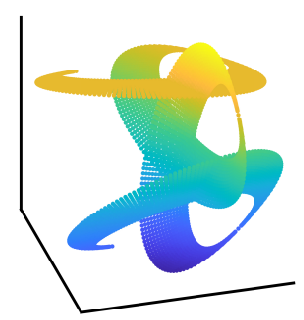

(b)
Fig. 13: (a) A sequence of $G^{1}$ PACSIC from dataset in 24, with 20 elastic parameters from 0.05 to 1 and with the stepsize 0.05. (b) A point cloud formed by a sequence of $G^{1}$ PACSIC from the same 5 points on a cube in Fig. 2, with 61 selections of the elastic parameters from -1.5 to 1.5 and stepsize 0.05 . 


\section{VIII. $G^{2}$ PACSIC}

Though the main focus of the paper is for $G^{1}$ PACSIC, it is natural to consider $G^{2}$ PACSIC. Different from $G^{1}$ scenario where each PCP is local, a $G^{2}$ PACSIC is global in general. Without going too far to the details, this section is dedicated to a brief description of $G^{2}$ PACSIC.

Lemma 1: With $\mathbf{B}_{1}(t)$ and $\mathbf{B}_{2}(t)$ in 7 , the $G^{2}$ continuity between $\mathbf{B}_{1}(t)$ and $\mathbf{B}_{2}(t)$ is equivalent to that all four elastic parameters $\beta_{1}, \alpha_{2}, \beta_{2}$ and $\alpha_{3}$ in $\mathbf{B}_{1}(t)$ and $\mathbf{B}_{2}(t)$ satisfy

$$
\begin{aligned}
& \frac{1}{\alpha_{2}^{2}}\left\|\left(\mathbf{P}_{3}-\mathbf{P}_{1}\right) \times\left(\beta_{1}\left(\mathbf{P}_{2}-\mathbf{P}_{0}\right)-\left(\mathbf{P}_{2}-\mathbf{P}_{1}\right)\right)\right\| \\
& =\frac{1}{\beta_{2}^{2}}\left\|\left(\mathbf{P}_{3}-\mathbf{P}_{1}\right) \times\left(\alpha_{3}\left(\mathbf{P}_{4}-\mathbf{P}_{2}\right)-\left(\mathbf{P}_{3}-\mathbf{P}_{2}\right)\right)\right\|,
\end{aligned}
$$

where $\mathbf{P}_{0}$ was assumed to be the vertex in front of $\mathbf{P}_{1}$, and $\mathbf{P}_{4}$ was assumed to be the vertex fter $\mathbf{P}_{3}$.

Proof: Denote by $\operatorname{Curv}(\mathbf{F})(t)$ the curvature of a curve $\mathbf{F}(t)$ at $t$. The $G^{2}$ condition between $\mathbf{B}_{1}(t)$ and $\mathbf{B}_{2}(t)$ is the curvature of $\operatorname{Curv}\left(\mathbf{B}_{1}\right)(1)=\operatorname{Curv}\left(\mathbf{B}_{2}\right)(0)$, i.e.,

$$
\left.\frac{\left\|\mathbf{B}_{1}^{\prime} \times \mathbf{B}_{1}^{\prime \prime}\right\|}{\left\|\mathbf{B}_{1}^{\prime}\right\|^{3}}\right|_{t=1}=\left.\frac{\left\|\mathbf{B}_{2}^{\prime} \times \mathbf{B}_{2}^{\prime \prime}\right\|}{\left\|\mathbf{B}_{2}^{\prime}\right\|^{3}}\right|_{t=0} .
$$

Due to the facts that

$$
\begin{aligned}
& \mathbf{B}_{1}^{\prime}(1)=3\left(\mathbf{P}_{2}-\mathbf{Q}_{1,2}\right), \mathbf{B}_{1}^{\prime \prime}(1)=6\left(\mathbf{Q}_{1,1}-2 \mathbf{Q}_{1,2}+\mathbf{P}_{2}\right), \\
& \mathbf{B}_{2}^{\prime}(0)=3\left(\mathbf{Q}_{2,1}-\mathbf{P}_{2}\right), \mathbf{B}_{2}^{\prime \prime}(0)=6\left(\mathbf{P}_{2}-2 \mathbf{Q}_{2,1}+\mathbf{Q}_{2,2}\right),
\end{aligned}
$$

and by using (8)-(9), the equality (26) leads to

$$
\begin{aligned}
& \mathbf{B}_{1}^{\prime}(1) \times \mathbf{B}_{1}^{\prime \prime}(1) \\
& =18\left(\mathbf{P}_{2}-\mathbf{Q}_{1,2}\right) \times\left(\mathbf{Q}_{1,1}-2 \mathbf{Q}_{1,2}+\mathbf{P}_{2}\right) \\
& \left.=18 \alpha_{2}\left(\mathbf{P}_{3}-\mathbf{P}_{1}\right) \times\left(-\beta_{1} \mathbf{P}_{0}+\mathbf{P}_{1}-\left(1-\beta_{1}\right) \mathbf{P}_{2}\right)\right) \\
& =18 \alpha_{2}\left(\mathbf{P}_{3}-\mathbf{P}_{1}\right) \times\left(\beta_{1}\left(\mathbf{P}_{2}-\mathbf{P}_{0}\right)-\left(\mathbf{P}_{2}-\mathbf{P}_{1}\right)\right) .
\end{aligned}
$$

Similarly,

$$
\begin{aligned}
& \mathbf{B}_{2}^{\prime}(0) \times \mathbf{B}_{2}^{\prime \prime}(0) \\
& =18 \beta_{2}\left(\mathbf{P}_{3}-\mathbf{P}_{1}\right) \times\left(\alpha_{3}\left(\mathbf{P}_{4}-\mathbf{P}_{2}\right)-\left(\mathbf{P}_{3}-\mathbf{P}_{2}\right)\right) .
\end{aligned}
$$

Together with $\left\|\mathbf{B}_{1}^{\prime}(1)\right\|^{3}=27\left|\alpha_{2}\right|^{3}\left\|\mathbf{P}_{3}-\mathbf{P}_{1}\right\|^{3}$ and $\left\|\mathbf{B}_{2}^{\prime}(0)\right\|^{3}=27\left|\beta_{2}\right|^{3}\left\|\mathbf{P}_{3}-\mathbf{P}_{1}\right\|^{3}, 25$ is arrived.

By introducing

$$
\begin{array}{lll}
\mathbf{d}_{1}=\mathbf{P}_{2}-\mathbf{P}_{1}, & \mathbf{d}_{2}=\mathbf{P}_{3}-\mathbf{P}_{2}, & \text { and } \\
\mathbf{e}_{0}=\mathbf{P}_{2}-\mathbf{P}_{0}, & \mathbf{e}_{1}=\mathbf{P}_{3}-\mathbf{P}_{1}, & \mathbf{e}_{2}=\mathbf{P}_{4}-\mathbf{P}_{2},
\end{array}
$$

$\operatorname{Curv}\left(\mathbf{B}_{1}\right)(1)$ and $\operatorname{Curv}\left(\mathbf{B}_{2}\right)(0)$ can be expressed by

$$
\begin{aligned}
& \operatorname{Curv}\left(\mathbf{B}_{1}\right)(1)=\frac{2}{3 \alpha_{2}^{2}} \frac{\left\|\mathbf{e}_{1} \times\left(\beta_{1} \mathbf{e}_{0}-\mathbf{d}_{1}\right)\right\|}{\left\|\mathbf{e}_{1}\right\|^{3}}, \\
& \operatorname{Curv}\left(\mathbf{B}_{2}\right)(0)=\frac{2}{3 \beta_{2}^{2}} \frac{\left\|\mathbf{e}_{1} \times\left(\alpha_{3} \mathbf{e}_{2}-\mathbf{d}_{2}\right)\right\|}{\left\|\mathbf{e}_{1}\right\|^{3}} .
\end{aligned}
$$

Moreover, 25] is equivalent to

$$
\begin{aligned}
\beta_{2}^{4} & {\left[\beta_{1}^{2}\left(\left\|\mathbf{e}_{0}\right\|^{2}\left\|\mathbf{e}_{1}\right\|^{2}-\left(\mathbf{e}_{0}^{\top} \mathbf{e}_{1}\right)^{2}\right)\right.} \\
& +2 \beta_{1}\left(-\left\|\mathbf{e}_{1}\right\|^{2} \mathbf{d}_{1}^{\top} \mathbf{e}_{0}+\mathbf{d}_{1}^{\top} \mathbf{e}_{1} \mathbf{e}_{0}^{\top} \mathbf{e}_{1}\right) \\
& \left.+\left\|\mathbf{d}_{1}\right\|^{2}\left\|\mathbf{e}_{1}\right\|^{2}-\left(\mathbf{d}_{1}^{\top} \mathbf{e}_{1}\right)^{2}\right] \\
= & \alpha_{2}^{4}\left[\alpha_{3}^{2}\left(\left\|\mathbf{e}_{1}\right\|^{2}\left\|\mathbf{e}_{2}\right\|^{2}-\left(\mathbf{e}_{1}^{\top} \mathbf{e}_{2}\right)^{2}\right)\right. \\
& +2 \alpha_{3}\left(-\left\|\mathbf{e}_{1}\right\|^{2} \mathbf{d}_{2}^{\top} \mathbf{e}_{2}+\mathbf{d}_{2}^{\top} \mathbf{e}_{1} \mathbf{e}_{1}^{\top} \mathbf{e}_{2}\right) \\
& \left.+\left\|\mathbf{d}_{2}\right\|^{2}\left\|\mathbf{e}_{1}\right\|^{2}-\left(\mathbf{d}_{2}^{\top} \mathbf{e}_{1}\right)^{2}\right] .
\end{aligned}
$$

In fact,

$$
\begin{aligned}
\| & \left(\mathbf{P}_{3}-\mathbf{P}_{1}\right) \times\left(\beta_{1}\left(\mathbf{P}_{2}-\mathbf{P}_{0}\right)-\left(\mathbf{P}_{2}-\mathbf{P}_{1}\right)\right) \|^{2} \\
= & \left\|\mathbf{e}_{1} \times\left(\beta_{1} \mathbf{e}_{0}-\mathbf{d}_{1}\right)\right\|^{2} \\
= & \left\|\mathbf{e}_{1}\right\|^{2}\left\|\beta_{1} \mathbf{e}_{0}-\mathbf{d}_{1}\right\|^{2}-\left(\mathbf{e}_{1} \cdot\left(\beta_{1} \mathbf{e}_{0}-\mathbf{d}_{1}\right)\right)^{2} \\
= & \left\|\mathbf{e}_{1}\right\|^{2}\left(\beta_{1} \mathbf{e}_{0}^{\top}-\mathbf{d}_{1}^{\top}\right)\left(\beta_{1} \mathbf{e}_{0}-\mathbf{d}_{1}\right) \\
& \left.\quad-\left(\beta_{1} \mathbf{e}_{1} \cdot \mathbf{e}_{0}-\mathbf{e}_{1} \cdot \mathbf{d}_{1}\right)\right)^{2} \\
= & \beta_{1}^{2}\left(\left\|\mathbf{e}_{0}\right\|^{2}\left\|\mathbf{e}_{1}\right\|^{2}-\left(\mathbf{e}_{0}^{\top} \mathbf{e}_{1}\right)^{2}\right) \\
& +2 \beta_{1}\left(-\left\|\mathbf{e}_{1}\right\|^{2} \mathbf{d}_{1}^{\top} \mathbf{e}_{0}+\mathbf{d}_{1}^{\top} \mathbf{e}_{1} \mathbf{e}_{0}^{\top} \mathbf{e}_{1}\right) \\
& +\left\|\mathbf{d}_{1}\right\|^{2}\left\|\mathbf{e}_{1}\right\|^{2}-\left(\mathbf{d}_{1}^{\top} \mathbf{e}_{1}\right)^{2},
\end{aligned}
$$

and, similarly,

$$
\begin{aligned}
& \left\|\left(\mathbf{P}_{3}-\mathbf{P}_{1}\right) \times\left(\alpha_{3}\left(\mathbf{P}_{4}-\mathbf{P}_{2}\right)-\left(\mathbf{P}_{3}-\mathbf{P}_{2}\right)\right)\right\|^{2} \\
& =\alpha_{3}^{2}\left(\left\|\mathbf{e}_{1}\right\|^{2}\left\|\mathbf{e}_{2}\right\|^{2}-\left(\mathbf{e}_{1}^{\top} \mathbf{e}_{2}\right)^{2}\right) \\
& \quad+2 \alpha_{3}\left(-\left\|\mathbf{e}_{1}\right\|^{2} \mathbf{d}_{2}^{\top} \mathbf{e}_{2}+\mathbf{d}_{2}^{\top} \mathbf{e}_{1} \mathbf{e}_{1}^{\top} \mathbf{e}_{2}\right) \\
& \quad+\left\|\mathbf{d}_{2}\right\|^{2}\left\|\mathbf{e}_{1}\right\|^{2}-\left(\mathbf{d}_{2}^{\top} \mathbf{e}_{1}\right)^{2} .
\end{aligned}
$$

In particular, when it is $2 \mathrm{~d}$, the sufficient conditions of both sides of (25) to be zero give rise to

$$
\begin{aligned}
\beta_{1} & =\frac{\left\|\mathbf{e}_{1}\right\|^{2} \mathbf{d}_{1}^{\top} \mathbf{e}_{0}-\mathbf{d}_{1}^{\top} \mathbf{e}_{1} \mathbf{e}_{0}^{\top} \mathbf{e}_{1}}{\left\|\mathbf{e}_{1}\right\|^{2}\left\|\mathbf{e}_{0}\right\|^{2}-\left(\mathbf{e}_{1}^{\top} \mathbf{e}_{0}\right)^{2}}, \\
\alpha_{3} & =\frac{\left\|\mathbf{e}_{1}\right\|^{2} \mathbf{d}_{2}^{\top} \mathbf{e}_{2}-\mathbf{d}_{2}^{\top} \mathbf{e}_{1} \mathbf{e}_{1}^{\top} \mathbf{e}_{2}}{\left\|\mathbf{e}_{1}\right\|^{2}\left\|\mathbf{e}_{2}\right\|^{2}-\left(\mathbf{e}_{1}^{\top} \mathbf{e}_{2}\right)^{2}},
\end{aligned}
$$

provided both denominators in (30)-31) are nontrivial. Fig. 14a shows a $2 \mathrm{~d} G^{2}$ PACSIC for the same PLF in Fig. 6 Fig. $14 \mathrm{~b}$ is another $2 \mathrm{~d} G^{2}$ PACSIC from a square with vertices $\{[1,0],[0,1],[-1,0],[0,-1]\}$. Nevertheless, formulations in (30)-31) were not applicable to the dataset in 24, as the denominators are zeros. The $G^{2}$ conditions in 25 for any PLF may result in numerous solutions for elastic parameters. Fig. $14 \mathrm{c}$ and Fig. $14 \mathrm{~d}$ are two $2 \mathrm{~d} G^{2}$ PACSIC from the same dataset in (24). However, even with some additional symmetric conditions imposed, solutions for elastic parameters, satisfying a system of nonlinear equations as in 25, are plenty. E.g., one family of the elastic parameters are given by

$$
\begin{aligned}
& {\left[\begin{array}{l}
\alpha_{1} \\
\beta_{1}
\end{array}\right]=\left[\begin{array}{l}
\alpha_{4} \\
\beta_{4}
\end{array}\right]=\left[\begin{array}{l}
\alpha_{7} \\
\beta_{7}
\end{array}\right]=\left[\begin{array}{l}
\alpha_{10} \\
\beta_{10}
\end{array}\right]=\left[\begin{array}{c}
\frac{\sin \theta}{1+\sin \theta} \\
\tan \theta
\end{array}\right],} \\
& {\left[\begin{array}{l}
\alpha_{2} \\
\beta_{2}
\end{array}\right]=\left[\begin{array}{l}
\alpha_{5} \\
\beta_{5}
\end{array}\right]=\left[\begin{array}{l}
\alpha_{8} \\
\beta_{8}
\end{array}\right]=\left[\begin{array}{l}
\alpha_{11} \\
\beta_{11}
\end{array}\right]=\frac{1}{1+\sin \theta}\left[\begin{array}{l}
1 \\
1
\end{array}\right],} \\
& {\left[\begin{array}{l}
\alpha_{3} \\
\beta_{3}
\end{array}\right]=\left[\begin{array}{l}
\alpha_{6} \\
\beta_{6}
\end{array}\right]=\left[\begin{array}{l}
\alpha_{9} \\
\beta_{9}
\end{array}\right]=\left[\begin{array}{l}
\alpha_{11} \\
\beta_{12}
\end{array}\right]=\left[\begin{array}{c}
\tan \theta \\
\sin \theta \\
1+\sin \theta
\end{array}\right] \text {, }}
\end{aligned}
$$


while another family are enumerated by

$$
\begin{aligned}
& {\left[\begin{array}{l}
\alpha_{1} \\
\beta_{1}
\end{array}\right]=\left[\begin{array}{l}
\alpha_{4} \\
\beta_{4}
\end{array}\right]=\left[\begin{array}{l}
\alpha_{7} \\
\beta_{7}
\end{array}\right]=\left[\begin{array}{l}
\alpha_{10} \\
\beta_{10}
\end{array}\right]=\left[\begin{array}{c}
\frac{\sin \theta}{1+\sin \theta} \\
\tan \theta
\end{array}\right],} \\
& {\left[\begin{array}{l}
\alpha_{2} \\
\beta_{2}
\end{array}\right]=\left[\begin{array}{l}
\alpha_{5} \\
\beta_{5}
\end{array}\right]=\left[\begin{array}{l}
\alpha_{8} \\
\beta_{8}
\end{array}\right]=\left[\begin{array}{l}
\alpha_{11} \\
\beta_{11}
\end{array}\right]=\frac{\sin \theta}{1+\sin \theta}\left[\begin{array}{l}
1 \\
1
\end{array}\right] \text {, }} \\
& {\left[\begin{array}{l}
\alpha_{3} \\
\beta_{3}
\end{array}\right]=\left[\begin{array}{l}
\alpha_{6} \\
\beta_{6}
\end{array}\right]=\left[\begin{array}{l}
\alpha_{9} \\
\beta_{9}
\end{array}\right]=\left[\begin{array}{l}
\alpha_{11} \\
\beta_{12}
\end{array}\right]=\left[\begin{array}{c}
\tan \theta \\
\sin \theta \\
1+\sin \theta
\end{array}\right],}
\end{aligned}
$$

for $\theta \in(0, \pi / 4)$. The elastic parameters for the two $G^{2}$ PACSIC shown in Fig. $14 \mathrm{c}$ and Fig. $14 \mathrm{~d}$ are each with the selection of $\theta=\pi / 8$ in (32) and (33); and the curvature at each vertex, which is not zero. In fact, the elastic parameters in 32 and (33) yield the same curvature at $(0,0)$, which is $\cot ^{2} \theta /(3 \sqrt{2})$; while at $(1,0)$ the curvature is $(1+\sin \theta)^{2} /(3 \sqrt{2})$ and $(1+$ $\csc \theta)^{2} /(3 \sqrt{2})$, respectively.

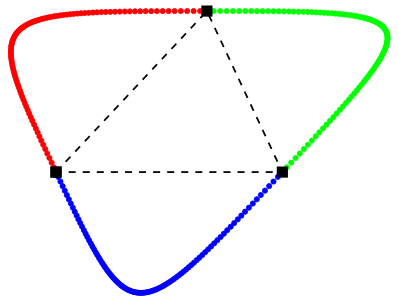

(a)

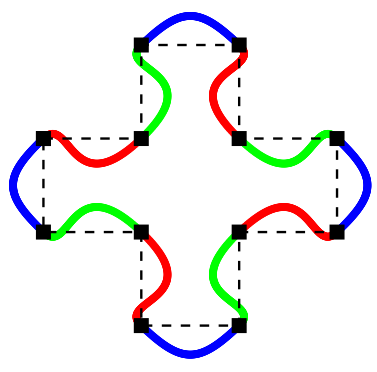

(c)

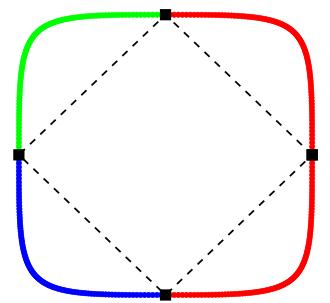

(b)

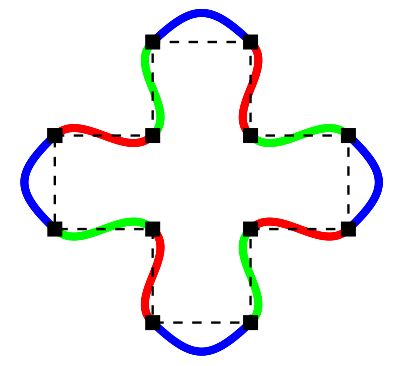

(d)
Fig. 14: (a) A 2d $G^{2}$ PACSIC from the same PLF in 20) (see both Fig. 5 and Fig. 6); (b) A $2 d G^{2}$ PACSIC for the PLF to be a unit square with vertices $\{[1,0],[0,1],[-1,0],[0,-1]\}$. The curvature at each vertex in both figures is actually zero. (c)-(d) Two slightly different $2 \mathrm{~d} G^{2}$ PACSIC for the PLF from the same dataset in 24, where the formulations in (30)-31 were not applicable; and all elastic parameters were listed in (32)- 33 , with the selection of $\theta=\pi / 8$.

\section{CONCLUSION}

Two algorithms for establishing PACSIC (parametric cubic spline interpolation curve) for any given dataset as an open or closed PLF (piecewise linear function) were established. Both algorithms do not require any prescribed tangent directions or any boundary conditions, though these directions can also be pre-defined if necessary. The direction at each vertex was simply determined by the two neighboring vertices. The LS (least square) $G^{1}$ PACSIC, in particular, can be applied to approximate any $2 \mathrm{~d}$ or $3 \mathrm{~d}$ dataset. The solution for the LS $G^{1}$ PACSIC is unique if each $i^{\text {th }}$ vertex is not equal to the $(i+2)^{\text {nd }}$ vertex. It also depends on the reference curve, which can be a line or a curve passing (or not) the two vertices. Simple examples were used to illustrate the flexibility of the algorithms, and various illustrative examples were also shown to demonstrate the efficiency of both algorithms. For completeness, the $G^{2}$ PACSIC was briefly investigated, where the $G^{2}$ conditions were given in terms of elastic parameters. Certainly, similar algorithms for generic PACSIC and LS PACSIC can also be developed if the tangent directions at vertices are evaluated by using three or more neighboring vertices; or even by raising the degree from cubic to quartic. Near future research will establish similar algorithms for surfaces, namely, PACSIS (parametric cubic spline interpolation surfaces) and their applications to other areas such as curve matching in image processing and feature extraction in data science.

\section{APPENDIX A \\ LEAST SQUARE BETWEEN A $G^{1}$ PACSIC AND A Line}

The notion of the square-integral for the surface formed by two parametric curves $\mathbf{F}_{1}(t)$ and $\mathbf{F}_{2}(t), t \in\left[t_{1}, t_{2}\right]$, is introduced by

$$
\int_{t_{1}}^{t_{2}}\left\|\mathbf{F}_{1}(t)-\mathbf{F}_{2}(t)\right\|^{2} d t .
$$

The formulations in Step 2 within Algorithm 2 in Section IV were consequences of the following.

Lemma 2: Let: (1) $\left\{\mathbf{x}_{1}, \mathbf{x}_{2}, \mathbf{x}_{3}, \mathbf{x}_{4}\right\}$ be four vectors (in $2 \mathrm{~d}$, $3 \mathrm{~d}$, or any dimensional); (2) $\mathbf{B}(t)$ be the PACSIC with one piece PCP determined by the four vectors $\mathbf{x}_{2}, \mathbf{y}_{2,1}, \mathbf{y}_{2,2}$, and $\mathbf{x}_{3}$, i.e.,

$$
\begin{aligned}
\mathbf{B}(t)=\mathbf{x}_{2} b_{3,0}(t)+\mathbf{y}_{2,1} b_{3,1}(t) & \\
& +\mathbf{y}_{2,2} b_{3,2}(t)+\mathbf{x}_{3} b_{3,3}(t), \quad t \in[0,1]
\end{aligned}
$$

where the two middle control points are in terms of $\mathbf{x}_{i}$ 's:

$$
\begin{aligned}
& \mathbf{y}_{2,1}=\mathbf{x}_{2}+b\left(\mathbf{x}_{3}-\mathbf{x}_{1}\right), \\
& \mathbf{y}_{2,2}=\mathbf{x}_{3}-a\left(\mathbf{x}_{4}-\mathbf{x}_{2}\right),
\end{aligned}
$$

for some constants $b$ and $a$; and (3) $\mathbf{L}(t)$ be the line passing $\mathbf{x}_{2}$ and $\mathbf{x}_{3}$, i.e.,

$$
\mathbf{L}(t)=\mathbf{x}_{2}(1-t)+\mathbf{x}_{3} t, \quad t \in[0,1] .
$$

$\mathbf{L}(t)$ in 34 is named as the reference curve. Then the minimization problem for the square-integral of the surface formed by two parametric curves

$$
\min _{b, a} \int_{0}^{1}\|\mathbf{B}(t)-\mathbf{L}(t)\|^{2} d t,
$$

has a unique solution for $b$ and $a$ if and only if

$$
\mathbf{x}_{1} \neq \mathbf{x}_{3}, \quad \mathbf{x}_{2} \neq \mathbf{x}_{4},
$$


with $b$ and $a$ being explicitly given by

$$
\begin{aligned}
& b=\frac{4\left\|\mathbf{v}_{2}\right\|^{2} \mathbf{v}_{1}^{\top} \mathbf{v}_{3}+3 \mathbf{v}_{2}^{\top} \mathbf{v}_{1} \mathbf{v}_{2}^{\top} \mathbf{v}_{3}}{3\left[16\left\|\mathbf{v}_{1}\right\|^{2}\left\|\mathbf{v}_{2}\right\|^{2}-9\left(\mathbf{v}_{1}^{\top} \mathbf{v}_{2}\right)^{2}\right]}, \\
& a=\frac{4\left\|\mathbf{v}_{1}\right\|^{2} \mathbf{v}_{2}^{\top} \mathbf{v}_{3}+3 \mathbf{v}_{1}^{\top} \mathbf{v}_{2} \mathbf{v}_{1}^{\top} \mathbf{v}_{3}}{3\left[16\left\|\mathbf{v}_{1}\right\|^{2}\left\|\mathbf{v}_{2}\right\|^{2}-9\left(\mathbf{v}_{1}^{\top} \mathbf{v}_{2}\right)^{2}\right]},
\end{aligned}
$$

where

$$
\mathbf{v}_{1}=\mathbf{x}_{3}-\mathbf{x}_{1}, \quad \mathbf{v}_{2}=\mathbf{x}_{4}-\mathbf{x}_{2}, \quad \mathbf{v}_{3}=\mathbf{x}_{3}-\mathbf{x}_{2} .
$$

Proof: Introduce

$$
\begin{aligned}
& \mathbf{F}(t)=\|\mathbf{B}(t)-\mathbf{L}(t)\|^{2}, \\
& \mathbf{G}(b, a)=\int_{0}^{1}\|\mathbf{B}(t)-\mathbf{L}(t)\|^{2} d t .
\end{aligned}
$$

Then

$$
\begin{aligned}
& \frac{1}{2} \frac{\partial}{\partial b} \mathbf{F}(t) \\
& =\left(\frac{\partial}{\partial b}\left(\mathbf{B}^{\top}(t)-\mathbf{L}^{\top}(t)\right)\right)(\mathbf{B}(t)-\mathbf{L}(t)) \\
& =\left(\mathbf{x}_{3}^{\top}-\mathbf{x}_{1}^{\top}\right) b_{3,1}(t)(\mathbf{B}(t)-\mathbf{L}(t)) \\
& =\left(\mathbf{x}_{3}^{\top}-\mathbf{x}_{1}^{\top}\right) b_{3,1}(t)\left[\mathbf{x}_{2} b_{3,0}(t)\right. \\
& \quad+\left(\mathbf{x}_{2}+b\left(\mathbf{x}_{3}-\mathbf{x}_{1}\right)\right) b_{3,1}(t) \\
& \quad+\left(\mathbf{x}_{3}-a\left(\mathbf{x}_{4}-\mathbf{x}_{2}\right)\right) b_{3,2}(t)+\mathbf{x}_{3} b_{3,3}(t) \\
& \left.\quad-\mathbf{x}_{2}(1-t)-\mathbf{x}_{3} t\right] \\
& =b\left\|\mathbf{v}_{1}\right\|^{2} b_{3,1}^{2}(t)-a \mathbf{v}_{1}^{\top} \mathbf{v}_{2} b_{3,1}(t) b_{3,2}(t) \\
& \quad+\mathbf{v}_{1}^{\top} \mathbf{x}_{2}\left[b_{3,0}(t)+b_{3,1}(t)-(1-t)\right] b_{3,1}(t) \\
& \quad+\mathbf{v}_{1}^{\top} \mathbf{x}_{3}\left[b_{3,2}(t)+b_{3,3}(t)-t\right] b_{3,1}(t)
\end{aligned}
$$

where $\mathbf{v}_{1}, \mathbf{v}_{2}$, and $\mathbf{v}_{3}$ in 39 ) were used. Similarly,

$$
\begin{aligned}
\frac{1}{2} \frac{\partial}{\partial a} & \mathbf{F}(t) \\
=- & b \mathbf{v}_{2}^{\top} \mathbf{v}_{1} b_{3,1}(t) b_{3,2}(t)+a\left\|\mathbf{v}_{2}\right\|^{2} b_{3,2}^{2}(t) \\
& \quad-\mathbf{v}_{2}^{\top} \mathbf{x}_{2}\left[b_{3,0}(t)+b_{3,1}(t)-(1-t)\right] b_{3,2}(t) \\
& \quad-\mathbf{v}_{2}^{\top} \mathbf{x}_{3}\left[b_{3,2}(t)+b_{3,3}(t)-t\right] b_{3,2}(t) .
\end{aligned}
$$

The simple facts

$$
\begin{aligned}
& \int_{0}^{1} b_{3,1}^{2}(t) d t=\int_{0}^{1} b_{3,2}^{2}(t) d t=\frac{3}{35}, \\
& \int_{0}^{1} b_{3,1}(t) b_{3,2}(t) d t=\frac{9}{140}, \\
& \int_{0}^{1}\left[b_{3,0}(t)+b_{3,1}(t)-(1-t)\right] b_{3,1}(t) d t \\
& =\int_{0}^{1}\left[b_{3,2}(t)+b_{3,3}(t)-t\right] b_{3,2}(t) d t=\frac{1}{140}, \\
& \int_{0}^{1}\left[b_{3,0}(t)+b_{3,1}(t)-(1-t)\right] b_{3,2}(t) d t \\
& =\int_{0}^{1}\left[b_{3,2}(t)+b_{3,3}(t)-t\right] b_{3,1}(t) d t=-\frac{1}{140}
\end{aligned}
$$

lead to

$$
\begin{aligned}
& \frac{1}{2} \frac{\partial}{\partial b} \mathbf{G}(b, a)=\int_{0}^{1} \frac{1}{2} \frac{\partial}{\partial b} \mathbf{F}(t) d t \\
& =\frac{3}{35} b\left\|\mathbf{v}_{1}\right\|^{2}-\frac{9}{140} a \mathbf{v}_{1}^{\top} \mathbf{v}_{2}-\frac{1}{140} \mathbf{v}_{1}^{\top} \mathbf{v}_{3} \\
& \frac{1}{2} \frac{\partial}{\partial a} \mathbf{G}(b, a)=\int_{0}^{1} \frac{1}{2} \frac{\partial}{\partial a} \mathbf{F}(t) d t \\
& =-\frac{9}{140} b \mathbf{v}_{2}^{\top} \mathbf{v}_{1}+\frac{3}{35} a\left\|\mathbf{v}_{2}\right\|^{2}-\frac{1}{140} \mathbf{v}_{2}^{\top} \mathbf{v}_{3} .
\end{aligned}
$$

Hence, the system of two linear equations $\frac{\partial}{\partial b} \mathbf{G}(b, a)=0$ and $\frac{\partial}{\partial a} \mathbf{G}(b, a)=0$ is equivalent to

$$
\left[\begin{array}{cc}
\frac{3}{35}\left\|\mathbf{v}_{1}\right\|^{2} & -\frac{9}{140} \mathbf{v}_{1}^{\top} \mathbf{v}_{2} \\
-\frac{9}{140} \mathbf{v}_{2}^{\top} \mathbf{v}_{1} & \frac{3}{35}\left\|\mathbf{v}_{2}\right\|^{2}
\end{array}\right]\left[\begin{array}{l}
b \\
a
\end{array}\right]=\frac{1}{140}\left[\begin{array}{c}
\mathbf{v}_{1}^{\top} \mathbf{v}_{3} \\
\mathbf{v}_{2}^{\top} \mathbf{v}_{3}
\end{array}\right],
$$

which gives the unique solution for $b$ and $a$ in (37)(38). Finally, the denominator in (37)- $(38)$ is nonnegative as $\left\|\mathbf{v}_{1}\right\|^{2}\left\|\mathbf{v}_{2}\right\|^{2} \geq\left(\mathbf{v}_{1}^{\top} \mathbf{v}_{2}\right)^{2}$. It is not zero if and only if $\mathbf{x}_{1} \neq \mathbf{x}_{3}$ and $\mathbf{x}_{2} \neq \mathbf{x}_{4}$, or 36 .

\section{APPENDIX B}

Least Square between a $G^{1}$ PACSIC and any Curve

The interpolation line $\mathbf{L}(t)$ in 34 was used for a reference curve when the minimization problem 35 in Appendix A was formulated. Observe that the reference curve $\mathbf{L}(t)$ can in fact be replaced by any parametric curve $\mathbf{F}(t), t \in[0,1]$. In other words, Lemma 2 in Appendix A can be generalized in the following.

Lemma 3: With the same assumptions as Lemma 2 but with $\mathbf{L}(t)$ in (34) being replaced by any curve

$$
\mathbf{H}(t), \quad t \in[0,1],
$$

the minimization problem

$$
\min _{b, a} \int_{0}^{1}\|\mathbf{B}(t)-\mathbf{H}(t)\|^{2} d t,
$$

has a unique solution for $b$ and $a$ if and only if 36 and with $b$ and $a$ being explicitly given by

$$
\begin{aligned}
& b=\frac{N_{1}}{3\left[16\left\|\mathbf{v}_{1}\right\|^{2}\left\|\mathbf{v}_{2}\right\|^{2}-9\left(\mathbf{v}_{1}^{\top} \mathbf{v}_{2}\right)^{2}\right]}, \\
& a=\frac{N_{2}}{3\left[16\left\|\mathbf{v}_{1}\right\|^{2}\left\|\mathbf{v}_{2}\right\|^{2}-9\left(\mathbf{v}_{1}^{\top} \mathbf{v}_{2}\right)^{2}\right]},
\end{aligned}
$$

where

$$
\begin{aligned}
& N_{1}=-140\left\|\mathbf{v}_{1}\right\|^{2}\left\|\mathbf{v}_{2}\right\|^{2}+88\left\|\mathbf{v}_{2}\right\|^{2} \mathbf{v}_{1}^{\top} \mathbf{v}_{3}-140\left\|\mathbf{v}_{2}\right\|^{2} \mathbf{v}_{1}^{\top} \mathbf{x}_{1} \\
& \quad+105\left(\mathbf{v}_{1}^{\top} \mathbf{v}_{2}\right)^{2}-39 \mathbf{v}_{1}^{\top} \mathbf{v}_{2} \mathbf{v}_{2}^{\top} \mathbf{v}_{3}+105 \mathbf{v}_{1}^{\top} \mathbf{v}_{2} \mathbf{v}_{2}^{\top} \mathbf{x}_{1} \\
& \quad+560\left\|\mathbf{v}_{2}\right\|^{2} \mathbf{v}_{1}^{\top} \mathbf{K}_{1}-420 \mathbf{v}_{1}^{\top} \mathbf{v}_{2} \mathbf{v}_{2}^{\top} \mathbf{K}_{2} \\
& N_{2}=140\left\|\mathbf{v}_{1}\right\|^{2} \mathbf{v}_{1}^{\top} \mathbf{v}_{2}-52\left\|\mathbf{v}_{1}\right\|^{2} \mathbf{v}_{2}^{\top} \mathbf{v}_{3}+140\left\|\mathbf{v}_{1}\right\|^{2} \mathbf{v}_{2}^{\top} \mathbf{x}_{1} \\
& \quad-105\left\|\mathbf{v}_{1}\right\|^{2} \mathbf{v}_{1}^{\top} \mathbf{v}_{2}+66 \mathbf{v}_{1}^{\top} \mathbf{v}_{2} \mathbf{v}_{1}^{\top} \mathbf{v}_{3}-105 \mathbf{v}_{1}^{\top} \mathbf{v}_{2} \mathbf{v}_{1}^{\top} \mathbf{x}_{1} \\
& \quad-560\left\|\mathbf{v}_{1}\right\|^{2} \mathbf{v}_{2}^{\top} \mathbf{K}_{2}+420 \mathbf{v}_{1}^{\top} \mathbf{v}_{2} \mathbf{v}_{1}^{\top} \mathbf{K}_{1}
\end{aligned}
$$




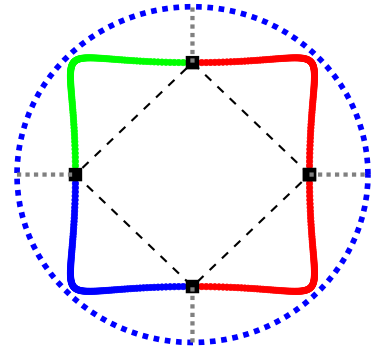

(a)

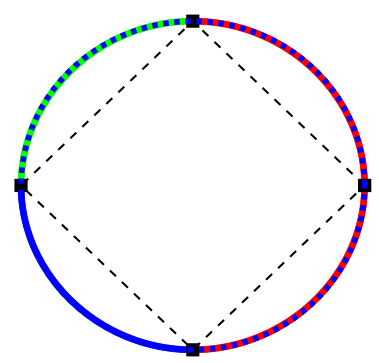

(b)

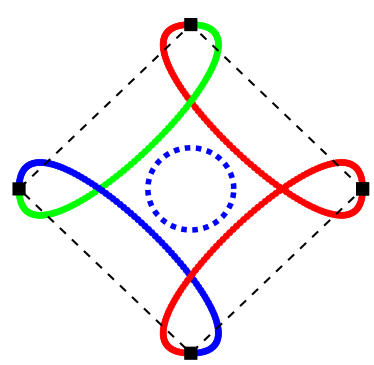

(c)

Fig. 15: The LS $G^{1}$ PACSIC from the dataset of a unit square and the reference curves as three quarterly partitioned circles with radii (a) 1.5 ; (b) 1 ; and (c) 0.25 .

$\mathbf{v}_{1}, \mathbf{v}_{2}$, and $\mathbf{v}_{3}$ are, again, in 39); and $\mathbf{K}_{1}$ and $\mathbf{K}_{2}$ are being evaluated from $\mathbf{H}$ through

$$
\mathbf{K}_{1}=\int_{0}^{1} b_{3,1}(t) \mathbf{H}(t) d t, \quad \mathbf{K}_{2}=\int_{0}^{1} b_{3,2}(t) \mathbf{H}(t) d t .
$$

The denominators for $a$ and $b$ in both Lemma 2 and Lemma 3 are the same; and Lemma 2 can also be considered as a consequence of Lemma 3 . Furthermore, the proof of Lemma 3 is completely analogous to the proof of Lemma 2 so it is omitted here.

Fig. 15 shows the LS $G^{1}$ PACSIC of the initial PLF as the same square used in Fig. 14b i.e., with vertices $\{[1,0],[0,1],[-1,0],[0,-1]\}$. Here the reference curves $\mathbf{F}(t)$ in Lemma 3 are circles with radii $1.5,1$, and 0.25 , which are quarterly partitioned. Notice that the unit circle and the 4-piece LS $G^{1}$ PACSIC in Fig. $15 b$ are nearly completely overlap. More precisely, the PACSIC in Quadrant I is the PLF with vertices $\left\{[1,0],\left[1,2 b_{1}\right],\left[2 a_{2}, 1\right],[0,1]\right\}$, where $b_{1}$ and $a_{2}$ are the elastic parameters which have the exact values

$$
b_{1}=a_{2}=\frac{560(\pi-3)}{\pi^{4}}-\frac{13}{24} .
$$

In other words, the cubic polynomial

$$
6 b_{1} t+3\left(1-4 b_{1}\right) t^{2}-2\left(1-3 b_{1}\right) t^{3}, \quad t \in[0,1],
$$

is a good approximation to the curve $\sin (\pi t / 2), t \in[0,1]$, where $b_{1}$ is in 407 .

\section{REFERENCES}

[1] M. Ammad And A. Ramli, Cubic B-spline curve interpolation with arbitrary derivatives on its data points, In: 2019 23rd International Conference in Information Visualization - Part II, 2019, pp. 156-159, doi: 10.1109/IV-2.2019.00038.

[2] M. Arunadevi, P. D. Jadhav, R. M. A, K. G. P, S. K. V and V. P. S, Machine learning approach for inverse kinematics in trajectory planning of pioneer 2 manipulator with cubic spline interpolation, in: 2021 5th International Conference on Computing Methodologies and Communication (ICCMC), 2021, pp. 807-813, doi: 10.1109/ICCMC51019.2021.9418318.

[3] C. DE Boor, A Practical Guide to Splines, Revised Ed., Applied Mathematical Sciences \#27, Springer, 2007

[4] G. Chaikin, An algorithm for high speed curve generation, Computer Graphics and Image Processing, vol. 3, 1974, pp. 346-349, https://doi.org/10.1016/0146-664X(74)90028-8.

[5] C.K. Chui, Multivariate Splines, CBMS-NSF Regional Conference Series \#54, SIAM, Philadelphia, 1988.

[6] G. CRISTESCU AND V. -F. DRĂGOI, Efficient approximation of two-terminal networks reliability polynomials using cubic splines, IEEE Transactions on Reliability70(3) (2021), pp. 1193-1203, doi: 10.1109/TR.2021.3049957.

[7] S. Ghosh AND D. P. Agrawal, Optimizing the energy envelope in the Internet of Things using predictive cubic splines, in: 2020 IEEE International Conference on Sensing, Communication and Networking (SECON Workshops), 2020, pp. 1-6, doi: 10.1109/SECONWorkshops50264.2020.9149775.

[8] S.-H. Hong, L. WANG AND T.-K. TRUONG, A new simple direct computation of cubic convolution spline interpolation, in: 2020 IEEE International Conference on Image Processing (ICIP), 2020, pp. 513517, doi: 10.1109/ICIP40778.2020.9191348.

[9] U. Khamdamov, D. Sultanov and M. Mukhiddinov, Application of digital signal processors based on methods of spline-functions for one-dimensional signals processing, in: 2020 International Conference on Information Science and Communications Technologies (ICISCT), 2020, pp. 1-4, doi: 10.1109/ICISCT50599.2020.9351509.

[10] S.P. MARTIN, An approach to data parametrization in parametric cubic spline interpolation problems, Journal of Approximation Theory, vol. 41, 1984, pp. 64-81, https://doi.org/10.1016/0021-9045(84)90121-7.

[11] C. A. MicChelli, Interpolatory subdivision schemes and wavelets, Journal of Approximation Theory, vol. 86, no. 1, pp. 41-71, https://doi.org/10.1006/jath.1996.0054.

[12] S. Okaniwa, A. Nasri, H. Lin, A. Abbas, Y. Kineri and T. MAEKAWA, Uniform B-spline curve interpolation with prescribed tangent and curvature vectors, IEEE Transactions on Visualization and Computer Graphics, vol. 18, no. 9, pp. 1474-1487, Sept. 2012, doi: 10.1109/TVCG.2011.262

[13] M. PANDEY, Futuristic hybrid Image enhancement using fuzzy and cubic interpolation methods, in: 2021 International Congress of Advanced Technology and Engineering (ICOTEN), 2021, pp. 1-6, doi: 10.1109/ICOTEN52080.2021.9493446.

[14] H. Prautzsch, W. Boehm, and M. Paluszny, Bézier and B-Spline Techniques, Springer-Verlag Berlin Heldelberg, 2002.

[15] L.L. SchumakeR, Spline Functions: Basic Theory (Cambridge Mathematical Library), 3rd Edition, Cambridge University Press, 2007.

[16] M C. Stone And T. D. DeRose, A geometric characterization of parametric cubic curves, ACM Transactions on Graphics, vol. 8, no. 3, July 1989, pp. 147-163. https://doi.org/10.1145/77055.77056.

[17] JiangKuan Su, Nian CaI, ShaOWEi WENG AND HaN Wang, Image interpolation with prescribed tangent of cubic B-spline curve, in: 2014 IEEE International Conference on Consumer Electronics-China, 2014, pp. 1-2, doi: 10.1109/ICCE-China.2014.7029877.

[18] S. Hong, L. Wang, T. Truong, T. Lin and L. Wang, Novel approaches to the parametric cubic-spline interpolation, IEEE Transactions on Image Processing, vol. 22, no. 3, pp. 1233-1241, March 2013, doi: 10.1109/TIP.2012.2230009. 